\title{
The parabolic Anderson model with heavy-tailed potential
}

\author{
Peter Mörters
}

\begin{abstract}
The parabolic Anderson model is the Cauchy problem for the heat equation with random potential. It offers a case study for the effects that a random, or irregular, environment can have on a diffusion process. The main focus in the present survey is on phenomena that are due to a highly irregular potential, which we model by a spatially independent, identically distributed random field with heavy tails. Among the effects we discuss are random fluctuations in the growth of the total mass, localisation in the weak and almost sure sense, and ageing.
\end{abstract}

2010 Mathematics Subject Classification. Primary 60K37 Secondary 82C44.

Keywords. Anderson Hamiltonian, parabolic problem, intermittency, localization, aging, out of equilibrium, random disorder, random medium, heavy tail, polynomial tail, Pareto distribution, Poisson point process, scaling limit.

\section{The parabolic Anderson problem}

We consider the heat equation with random potential on the integer lattice $\mathbb{Z}^{d}$ and study the Cauchy problem with localised initial datum,

$$
\begin{aligned}
\partial_{t} u(t, z) & =\Delta u(t, z)+\xi(z) u(t, z), & & \text { for }(t, z) \in(0, \infty) \times \mathbb{Z}^{d}, \\
\lim _{t \downarrow 0} u(t, z) & =\mathbf{1}_{0}(z), & & \text { for } z \in \mathbb{Z}^{d},
\end{aligned}
$$

where

$$
(\Delta f)(z)=\sum_{\substack{y \in \mathbb{Z}^{d} \\|y-z|=1}}[f(y)-f(z)], \quad \text { for } z \in \mathbb{Z}^{d}, f: \mathbb{Z}^{d} \rightarrow \mathbb{R}
$$

is the discrete Laplacian, and the potential $\left(\xi(z): z \in \mathbb{Z}^{d}\right)$ is a collection of independent identically distributed random variables. This problem appears in the context of chemical kinetics and population dynamics, and also provides a simplified qualitative approach to problems in magnetism and turbulence. Its name of parabolic Anderson problem goes back to the work of the nobel-prize winning physicist P.W. Anderson on entrapment of electrons in crystals with impurities, see [An58]. The references [GM90], [Mo94] and [CM94] provide applications, background and heuristics around the parabolic Anderson model and its relatives. 
Interesting recent mathematical progress not discussed here can be found, for example, in [HKM06], [GKM07], [BMR07], [GHM07] and [D08], two survey articles emphasising recent work on a range of potentials are [GK05] and [GHM08]. Note that in some of these references the potential field is allowed to have a nontrivial time-dependence, a feature which we shall exclude from the discussions of the present paper.

The parabolic Anderson problem has a unique nonnegative solution if

$$
E\left[(\xi(0) \vee 0)^{d+\epsilon}\right]<\infty \text { for some } \epsilon>0,
$$

see [GM90]. Under this condition, the solution has a probabilistic representation known as the Feynman-Kac formula. Indeed, suppose the potential $\left(\xi(z): z \in \mathbb{Z}^{d}\right)$ is fixed and let $\left(X_{s}: s \geq 0\right)$ be a continuous time random walk with generator $\Delta$ started at the origin. Let a particle following this walk have a mass, which is initially set to one. Suppose the particle mass grows when the particle sits at a site $z$ with positive potential with rate $\xi(z)$, and shrinks when the particle sits at a site $z$ with nonpositive potential with rate $-\xi(z)$. The solution $u(t, z)$ of the parabolic Anderson problem is then given as the expected mass of particles at site $z$ at time $t$. In other words,

$$
u(t, z)=\mathbb{E}_{0}\left[\mathbf{1}_{\left\{X_{t}=z\right\}} \exp \left(\int_{0}^{t} \xi\left(X_{s}\right) d s\right)\right]
$$

where the expectation refers only to the random walk, so that the solution is random due to its dependence on the potential $\left(\xi(z): z \in \mathbb{Z}^{d}\right)$.

The main reason for the great interest the parabolic Anderson model has received over the past ten years is due to the intermittency effect which is believed to be present in the model as soon as the potential random variables $\xi(z)$ are truly random. Loosely speaking, intermittency means that as time progresses, the bulk of the mass of the solution is not spreading in a regular fashion, but becomes concentrated in a small number of spatially separated connected sets of moderate size, whose location is determined by the potential, which are called intermittent islands. This means that there is a marked contrast between the behaviour of a diffusion in a constant potential, which, by the central limit theorem, spreads the bulk of its mass at time $t$ over a ball of radius of order $\sqrt{t}$, and the behaviour of a random potential with even the slightest randomness. For example, in the case of a potential given by $P\{\xi(0)=0\}=\varepsilon, P\{\xi(0)=-\delta\}=1-\varepsilon$, for $\varepsilon, \delta>0$, Biskup and König [BK01] provide evidence that the mass is almost surely concentrated in a small number of islands with diameter of order $(\log t)^{1 / d}$ located in areas where the potential has a high concentration of zeroes.

On a heuristical level, the reason for this intermittent behaviour is the competition between the benefits of the random walk path spending much time at sites with large potential values, which is manifest from the exponential term in (2), and the unlikeliness of such paths. Even in the case of an only mildly random 
potential, there is an exponential advantage in spending most of the time in an area with maximal potential and thefore exponentially unlikely random walk paths make the dominant contribution to the expectation in (2). The strength of this effect depends on the distribution of the potential values $\xi(0)$, more precisely on the tail of the distribution of $\xi(0)$ at infinity. If the distribution has a bounded support, the main contribution will come from walks confinded to islands consisting of sites with near maximal potential values. There will be a careful balance between the probability that a random walk reaches such an island in time $o(t)$ and stays there for the remaining time on the one hand, and the height of the potential on the island on the other hand. We expect that as time progresses the walk can reach larger and larger islands. Such behaviour also prevails if $\xi(0)$ has a very light tail at infinity. If the upper tail of $\xi(0)$ is sufficiently heavy however, we expect that only random walks that go to certain optimal sites in time $o(t)$ and remain at such a site for almost the entire time will contribute to the expectation. In this case the solution is localised in islands which are single sites and, in particular, do not grow in time.

It is a very hard problem to make the above heuristics rigorous, confirm the geometric picture of intermittency and study the precise time-dependence of the size of the islands as a function of the distribution of the potential values. Worse even, hardly anything is known about the number of islands on which the solutions are concentrated. Apart from the work described here, we note that progress on the geometry of the solutions has been made on the one hand in the work of Sznitman for the closely related continuous model of a Brownian motion with Poissonian obstacles, the work of his group is surveyed in the monograph [Sz98], and on the other hand in the seminal paper of Gärtner et al. [GKM07], which treats the vicinity of the double-exponential distribution. The bulk of the literature however offers an alternative, less explicit, approach to the problem, by giving a rigorous expansion of the growth rate of the total mass of the solution in the time variable. This can then be interpreted in terms of geometric quantities like the size of the islands, and the height and profile of the solution on an island, see Section 2 for some more detail. In this context it was shown in [HKM06] that there are four universality classes in the parabolic Anderson model, dividing potentials into classes corresponding to qualitatively different types of intermittent behaviour.

Roughly speaking, we can learn from this analysis that we can expect islands to be growing over time if the tails are light enough to satisfy

(A) $\frac{1}{x} \log |\log \mathbb{P}\{\xi(0)>x\}| \longrightarrow \infty$ as $x \uparrow \infty$,

whereas the islands consist of single sites if

(B) $\frac{1}{x} \log |\log \mathbb{P}\{\xi(0)>x\}| \longrightarrow 0$ as $x \uparrow \infty$. 
Class (A) covers all bounded potentials and some incredibly light-tailed unbounded ones, most of the unbounded potentials (in particular the class of Gaussian potentials) belong to class (B). Note that the rigorous results about this classification require mild additional regularity assumptions, which we neglect for the purpose of this introduction.

The present paper reports on the progress obtained in the attempt to study the geometry of the solutions for potentials which lead to islands consisting of single sites. Apart from the author of this survey, the researchers involved in various stages of this project were Remco van der Hofstad (Eindhoven), Wolfgang König (Berlin), Hubert Lacoin (Paris), Marcel Ortgiese (Berlin) and Nadia Sidorova (London). For our analysis we chose the potentials with the heaviest possible tails. In fact we assumed that the potentials follow the Pareto distribution

$$
P\{\xi(0) \geq x\}=x^{-\alpha} \quad \text { for all } x \geq 1
$$

We assumed that the parameter $\alpha$ is strictly bigger than the lattice dimension $d$, which is necessary and sufficient for the existence of a nonnegative solution to the parabolic Anderson problem. While the choice of a potential with only polynomial decay at infinity was expected to make the possible qualitative effects of the random environment very pronounced, we were facing the technical challenge that much of the established techniques to study the parabolic Anderson problem were not available to us, as they require finiteness of some moments of the solution. As a result, new techniques had to be developed. I will give a flavour of these techniques when presenting the results of this project in the following sections.

\section{The growth rate of the total mass}

For comparison, we start by looking at the situation in the case of potential with milder irregularities, more precisely we assume that all exponential moments (with positive rate) of the random variable $\xi(0)$ are finite. Then the function

$$
H(t):=\log E e^{t \xi(0)}, \quad \text { for } t>0
$$

is well-defined and finite. Define

$$
U(t):=\sum_{z \in \mathbb{Z}^{d}} u(t, z)
$$

to be the total mass of the system at time $t$. A large deviation heuristic (detailed, for example, in [HKM06]) suggests that the following almost sure expansion holds, as $t \uparrow \infty$,

$$
\frac{1}{t} \log U(t)=\frac{H\left(\beta_{t} \alpha\left(\beta_{t}\right)^{-d}\right)}{\beta_{t} \alpha\left(\beta_{t}\right)^{-d}}-\alpha\left(\beta_{t}\right)^{2}(\kappa+o(1)),
$$


where $\alpha$ and $\beta$ are positive scale-functions and $\alpha\left(\beta_{t}\right)$ plays the rôle of the order of the diameter of the intermittent islands at time $t$. The first order term describes the height of the potential on an island at time $t$ and therefore the growth rate of the solution. The number $\kappa$ in the second order term is the minimiser in a variational problem whose optimiser describes the profile of the solution on an island scaled to diamater of constant order. As indicated above, the papers [GM98, BK01, HKM06] give rigorous asymptotic expansions of $\frac{1}{t} \log U(t)$ up to the second order term, which can then be interpreted in terms of these heuristics. Between them they cover all potentials with finite exponential moments, subject to mild regularity assumptions.

Note that the heuristics above predicts that the two leading terms in the expansion of the random variable $\frac{1}{t} \log U(t)$ are deterministic. In [HMS08] we have shown that this does not apply in the case of the Pareto potential, as already the leading term is random.

Theorem 2.1 (Weak asymptotics of the growth rate, Theorem 1.2 in [HMS08]). Suppose that the random variable $\xi(0)$ is Pareto distributed with parameter $\alpha>d$. Then, as $t \uparrow \infty$,

$$
\frac{(\log t)^{\frac{d}{\alpha-d}}}{t^{\frac{\alpha}{\alpha-d}}} \log U(t) \Rightarrow Y, \quad \text { where } \quad P\{Y \leq y\}=\exp \left(-\theta y^{d-\alpha}\right)
$$

and

$$
\theta:=\frac{(\alpha-d)^{d} 2^{d} B(\alpha-d, d)}{d^{d}(d-1) !}
$$

where $B$ denotes the beta function.

\section{Remark 2.2.}

- The limit law $Y$ is of extremal Fréchet type with shape parameter $\alpha-d$.

- The fact that already the first term in the expansion of $\frac{1}{t} \log U(t)$ is random is due to the extreme irregularity of the Pareto potentials. The main result of [HMS08] is an expansion for the case of the Weibull potentials, which shows that in this case the leading term in the expansion is still deterministic. Starting from the second term we have a discrepancy between the almost sure liminf and limsup behaviour. A weak limit theorem with nondegenerate limit distribution can only be observed for the fourth term, in which case we see a limit variable of Gumbel type.

We can also describe the fluctuations in the growth rate in an almost sure sense. To this end, we use the abbreviation

$$
L_{t}:=\frac{1}{t} \log U(t)
$$


Theorem 2.3 (Almost sure asymptotics of the growth rate, Theorem 1.1 in [HMS08]). Suppose that the random variable $\xi(0)$ is Pareto distributed with parameter $\alpha>d$. Then, almost surely,

$$
\begin{aligned}
& \limsup _{t \rightarrow \infty} \frac{\log L_{t}-\frac{d}{\alpha-d} \log t}{\log \log t}=-\frac{d-1}{\alpha-d}, \quad \text { for } d>1, \\
& \limsup _{t \rightarrow \infty} \frac{\log L_{t}-\frac{d}{\alpha-d} \log t}{\log \log \log t}=\frac{1}{\alpha-d}, \quad \text { for } d=1,
\end{aligned}
$$

and

$$
\liminf _{t \rightarrow \infty} \frac{\log L_{t}-\frac{d}{\alpha-d} \log t}{\log \log t}=-\frac{d}{\alpha-d}, \quad \text { for } d \geq 1 .
$$

Remark 2.4. Theorem 2.1 shows that the liminf above is indeed a limit in probability, which demonstrates that the differing limsup behaviour is due to a small number of exceptional time scales where the growth rate is slightly bigger than typical.

We postpone the discussion of proof techniques to Section 4 where we give a considerable strengthening of Theorem 2.1.

\section{Localisation: The one- and the two-cities theorem}

Having looked at the total mass $U(t)$, we now shift our interest to the profile of the solution defined as

$$
v(t, z):=\frac{u(t, z)}{U(t)} \quad \text { for } t>0, z \in \mathbb{Z}^{d} .
$$

In other words, for any time $t$, we define $v(t, z)$ as the proportion of mass allocated to the site $z$. Suppose that our potential is of class (B) and islands are expected to consist of single lattice sites. Apart from confirming this rigorously, the most interesting question in this situation is how many islands are required to support the bulk of the solution.

Question: Find $n=n(t)$ as small as possible such that, for suitable (pairwise distinct) random points $Z_{t}^{(1)}, \ldots, Z_{t}^{(n)} \in \mathbb{Z}^{d}$, we have

$$
\lim _{t \uparrow \infty} \sum_{i=1}^{n} v\left(t, Z_{t}^{(i)}\right)=1,
$$

where the limit could be (a) in probability, or (b) almost surely.

This problem is essentially open for all nontrivial potentials in class (B) except for the Pareto potential, where it is solved in [KLMS09], a result we now present. We suppose from this point on in all our theorems that the random variable $\xi(0)$ is Pareto distributed with parameter $\alpha>d$. 
Theorem 3.1 (One point localisation in probability, Theorem 1.2 in [KLMS09]). There exists a càdlàg process $\left(Z_{t}: t>0\right)$ with values in $\mathbb{Z}^{d}$, depending only on the potential field, such that

$$
\lim _{t \rightarrow \infty} v\left(t, Z_{t}\right)=1 \quad \text { in probability }
$$

\section{Remark 3.2.}

- The solution is concentrated in just one site with high probability, a phenomenon often called complete localisation. To the best of our knowledge this has not been observed in any lattice-based model of mathematical physics so far, but it is not uncommon in mean-field models, see, for example, [FG92, FM90].

- We conjecture that the one-point localisation phenomenon holds for a wider class of heavy-tailed potentials, including the Weibull potentials, but does not hold for all potentials in class (A). In particular, it would be interesting to learn whether in the case of exponential distributions one needs $n(t) \rightarrow \infty$ points to cover the bulk of the solution.

- An investigation of the proof of Theorem 2.1 given in [HMS08] shows that

$$
\frac{(\log t)^{\frac{d}{\alpha-d}}}{t^{\frac{\alpha}{\alpha-d}}} \log v\left(t, Z_{t}\right) \Longrightarrow 0 .
$$

Note that this together with the asymptotics in Theorem 2.1 does not yield the concentration property in Theorem 3.1 since the asymptotics are only logarithmic. Much more precise techniques are required to prove the full strength of Theorem 3.1 .

We describe the philosophy behind the proof, sketching the argument detailed in [KMS06]. To guess the right choice of $\left(Z_{t}: t \geq 0\right)$ assume for the moment that the competition between the paths contributing to the expectation

$$
U(t)=\mathbb{E}_{0}\left[\exp \left(\int_{0}^{t} \xi\left(X_{s}\right) d s\right)\right]
$$

is only between paths that go to a site $z$ in time $o(t)$ and stay there. While the exponential factor in this case yields $\exp (t \xi(z)(1+o(1))$, for $z$ sufficiently far away from the origin the probability of such a path is essentially given by the probability that a random walk makes the minimum number of steps required to reach site $z$, which is the $\ell^{1}$-norm $\|z\|_{1}$. As the number of steps of the walk in $t$ time units is a Poisson random variable with mean $2 d t$ the cost of reaching $z$ is approximately

$$
\frac{(2 d t)^{\|z\|_{1}}}{\|z\|_{1} !} e^{-2 d t}=\exp \left(-\|z\|_{1} \log \frac{\|z\|_{1}}{2 d t e}(1+o(1))\right) .
$$


Therefore we choose $Z_{t}$ as the maximiser of the function

$$
\Psi_{t}(z)=\xi(z)-\frac{\|z\|_{1}}{t} \log \frac{\|z\|_{1}}{2 d t e}, \quad \text { for } z \in \mathbb{Z}^{d} .
$$

Note that the subtracted 'penalty term' depends increasingly on the $\ell^{1}$-norm of the site $z$. Therefore, for the proof of Theorem 3.1, we can construct a centred $\ell^{1}$-ball of random, time-dependent radius $h_{t}$ so that $Z_{t}$ is the site of maximal potential value in that box. Note that $\left\|Z_{t}\right\|_{1}$ would be a possible choice of such a radius, but in fact we can typically make it a bit larger. Given a site $z$ and large time $t$ we split $u(t, z)$ into three terms, which correspond to the contributions to the Feynman-Kac formula coming from paths that

(1) by time $t$ have left the ball $\left\{z:\|z\|_{1} \leq h_{t}\right\}$,

(2) stay inside this ball up to time $t$ but do not visit $Z_{t}$, and

(3) stay inside this ball and do visit $Z_{t}$.

It turns out that the total mass of the first two terms is negligible. For the first contribution this comes from an analysis, based on extreme value techniques, that shows that the radius $h_{t}$ is very large at time $t$ with high probability, so that it is very unlikely for random walk paths to leave this ball before time $t$. The argument for the second term is based on the fact, also obtained from extreme value analysis, that with high probability there is a large gap between the largest and the secondlargest value of $\left\{\Psi_{t}(z): z \in \mathbb{Z}^{d}\right\}$. Hence the contribution of paths avoiding $Z_{t}$ is small compared to paths that spend a significant amount of time there.

It finally remains to show that there is only a negligible contribution from paths that stay in the box, visit $Z_{t}$ but do not end up in $Z_{t}$ at time $t$. The argument for this is based on a spectral analytical device, which is used in a similar manner as in [GKM07]: We show that the third term above can be controlled in terms of the principal eigenfunction of the Anderson Hamiltonian, $\Delta+\xi$, in the ball with zero boundary conditions. This eigenfunction turns out to be exponentially concentrated in the maximal potential point in the ball, which by construction is $Z_{t}$. Hence the total mass $U$ must be concentrated in $Z_{t}$, completing the sketch of the proof of Theorem 3.1.

Remark 3.3. The convergence in Theorem 3.1 cannot hold in the almost-sure sense. Indeed, assume that $v\left(t, Z_{t}\right)>2 / 3$ for all $t \geq t_{0}$. As $v(\cdot, z)$ is continuous for any $z \in \mathbb{Z}^{d}$, at any jump time $t \geq t_{0}$ of the process $\left(Z_{t}: t \geq 0\right)$ we have $v\left(t, Z_{t}-\right)+v\left(t, Z_{t}\right)>4 / 3$, a contradiction. From the growth of $U(t)$ one can see that $\left(Z_{t}: t \geq 0\right)$ is not eventually constant, and thus has jumps at arbitrarily large times.

By the previous remark, at least two sites are needed to carry the total mass in an almost sure limit theorem. The main result of [KLMS09] shows that, in the case of Pareto distributed potentials, we have indeed almost sure localisation of the solution $u(t, \cdot)$ in two distinct lattice points $Z_{t}^{(1)}$ and $Z_{t}^{(2)}$, as $t \rightarrow \infty$. 
Theorem 3.4 (Two cities theorem, Theorem 1.1 in [KLMS09]). There exist processes $\left(Z_{t}^{(1)}: t>0\right)$ and $\left(Z_{t}^{(2)}: t>0\right)$ with values in $\mathbb{Z}^{d}$, depending only on the potential field, such that $Z_{t}^{(1)} \neq Z_{t}^{(2)}$ for all $t>0$, and

$$
\lim _{t \rightarrow \infty} v\left(t, Z_{t}^{(1)}\right)+v\left(t, Z_{t}^{(2)}\right)=1 \quad \text { almost surely. }
$$

Remark 3.5. The term two cities theorem was suggested by S.A. Molchanov. The underlying intuition is that at a typical large time the mass, which is thought of as a population, inhabits one site, interpreted as a city. At some rare times, however, the entire population moves to the new city, so that at the transition times part of the population still lives in the old city, while part has already moved to the new one.

Again we sketch the philosophy behind the proof. The main reason why this result is much harder than Theorem 3.1 is that the approximation of $\frac{1}{t} \log U(t)$ by the maximum of $\Psi_{t}$ is not good enough at all large times $t$ and a more complex variational problem has to be built to describe the cost and benefit of paths spending their time predominantly at a site $z$.

To this end, we look at the event that, for some $\rho>0$, the random walk wanders directly to a site $z$ during the time interval $[0, \rho t]$ and stays there throughout $[\rho t, t]$. Denoting $\eta(z):=\log \#\left\{\right.$ paths of length $\|z\|_{1}$ from origin to $\left.z\right\}$, this event has probability

$$
\begin{aligned}
\frac{e^{\eta(z)}}{(2 d)^{\|z\|_{1}}} & \frac{(2 d \rho t)^{\|z\|_{1}}}{\|z\|_{1} !} e^{-2 d \rho t} e^{-2 d(1-\rho) t} \\
& =\exp \left(-\|z\|_{1} \log \frac{\|z\|_{1}}{\rho t e}-2 d t+\eta(z)+o(t)\right) .
\end{aligned}
$$

The reward for this behaviour is $\exp (t(1-\rho) \xi(z)(1+o(1)))$ and therefore we look at those $(\rho, z)$ which maximise

$$
\sup _{z \in \mathbb{Z}^{d}} \sup _{\rho \in(0,1)}\left\{(1-\rho) \xi(z)-\frac{\|z\|_{1}}{t} \log \frac{\|z\|_{1}}{\rho t e}+\frac{\eta(z)}{t}\right\}
$$

Looking for the global maximimiser of the inner variational problem we get $\rho=$ $\|z\|_{1} /(t \xi(z))$, which for large $t$ becomes smaller than 1 . Hence $Z_{t}^{(1)}$ and $Z_{t}^{(2)}$ are chosen as the two largest values of

$$
\Phi_{t}(z):=\xi(z)-\frac{\|z\|_{1}}{t} \log \xi(z)+\frac{\eta(z)}{t} .
$$

We are then able to show that, almost surely,

$$
\frac{1}{t} \log U(t) \sim \max _{z \in \mathbb{Z}^{d}} \Phi_{t}(z)
$$

The proof of Theorem 3.4 is, just as in the case of the one-point localisation, based on a decomposition of paths, this time five rather than three cases need to be 
distinguished, and a similar, albeit slightly refined, arsenal of techniques. Without going into detail, the big difference is that in the almost-sure sense we can only expect a gap between the largest and the third-largest value of $\left\{\Phi_{t}(z): z \in \mathbb{Z}^{d}\right\}$, because whenever $t_{0}$ is such that the maximiser $z_{1}$ for all large $t<t_{0}$ is different from the maximiser $z_{2}$ for all small $t>t_{0}$, we necessarily have $\Phi_{t}\left(z_{1}\right)=\Phi_{t}\left(z_{2}\right)$ by continuity of the mapping $t \mapsto \Phi_{t}(z)$. This requires a different treatment of cases where the gap is between the largest and second-largest value, or between the second-largest and third-largest value, respectively.

\section{Scaling limit theorems}

Having seen that the solution of the parabolic Anderson problem with Pareto distributed potential field is concentrated in a single point at most times, it is natural to ask how the location of that point moves as time progresses. Theorem 1.3 (a) of [MOS09] gives a functional scaling limit theorem for the localisation point, together with the value of the potential in that point. For definiteness of the formualtion define $X_{t}$ by the property that $v\left(t, X_{t}\right)$ is the maximum value of the profile at time $t$. For the (countably many) times where this peak is not unique we choose the one with the smallest $\ell^{1}$-norm.

Theorem 4.1 (Functional scaling limit theorem, Theorem 1.3 of [MOS09]).

There exists a time-inhomogeneous Markov process $\left(\left(Y_{t}^{(1)}, Y_{t}^{(2)}\right): t>0\right)$ on $\mathbb{R}^{d} \times \mathbb{R}$ such that, as $T \rightarrow \infty$, we have

$$
\begin{aligned}
\left(\left(\left(\frac{\log T}{T}\right)^{\frac{\alpha}{\alpha-d}} X_{t T},\left(\frac{\log T}{T}\right)^{\frac{d}{\alpha-d}} \xi\left(X_{t T}\right)\right): t>0\right) \\
\Longrightarrow\left(\left(Y_{t}^{(1)}, Y_{t}^{(2)}+\frac{d}{\alpha-d}\left\|Y_{t}^{(1)}\right\|_{1}\right): t>0\right),
\end{aligned}
$$

in distribution on the space of càdlàg functions $f:(0, \infty) \rightarrow \mathbb{R}^{d} \times \mathbb{R}$ with respect to the Skorokhod topology on compact subintervals of $(0, \infty)$.

Before we give a detailed description of the limiting process and comment on the proof of this result, we discuss some of its interesting consequences.

\section{Remark 4.2.}

- Projecting onto the first coordinate at time $t=1$ we obtain, as $T \rightarrow \infty$,

$$
\left(\frac{\log T}{T}\right)^{\frac{\alpha}{\alpha-d}} X_{T} \Rightarrow Y
$$

in distribution. This means that the contributing random walks move with superlinear speed to the optimal point, a very remarkable fact. This result was also obtained as Theorem 1.3 in [KLMS09] where the limiting random variable was characterised by its density

$$
p(x)=\alpha \int_{0}^{\infty} \frac{\exp \left(-\theta y^{d-\alpha}\right) \mathrm{d} y}{\left(y+\frac{d}{\alpha-d}\|x\|_{1}\right)},
$$

where $\theta$ is the same constant as in Theorem 2.1. 
- Using that $\frac{1}{t} \log U(t) \sim \max _{z \in \mathbb{Z}^{d}} \Psi_{t}(z)$ in probability, we can derive from Theorem 4.1 a functional version of Theorem 2.1: As $T \rightarrow \infty$, we have

$$
\left(\left(\frac{\log T}{T}\right)^{\frac{d}{\alpha-d}} \frac{\log U(t T)}{t T}: t>0\right) \Longrightarrow\left(Y_{t}^{(2)}+\frac{d}{\alpha-d}\left(1-\frac{1}{t}\right)\left\|Y_{t}^{(1)}\right\|_{1}: t>0\right) .
$$

As all involved processes are continuous, this convergence holds in distribution on the space of continuous functions $f:(0, \infty) \rightarrow \mathbb{R}$ with respect to the uniform topology on compact subintervals.

- To formulate a more classical (but weaker) scaling limit theorem we extend the profile to $(0, \infty) \times \mathbb{R}^{d}$ by taking the integer parts of the second coordinate, letting $v(t, x):=v(t,\lfloor x\rfloor)$. Taking nonnegative measurable functions on $\mathbb{R}^{d}$ as densities with respect to the Lebesgue measure, we can interpret $a^{d} v(t, a x)$ for any $a, t>0$ as an element of the space $\mathcal{M}\left(\mathbb{R}^{d}\right)$ of probability measures on $\mathbb{R}^{d}$. Denoting by $\delta(y) \in \mathcal{M}\left(\mathbb{R}^{d}\right)$ the Dirac point mass located in $y \in \mathbb{R}^{d}$ we obtain, as $T \uparrow \infty$,

$$
\left(\left(\frac{T}{\log T}\right)^{\frac{\alpha d}{\alpha-d}} v\left(t T,\left(\frac{T}{\log T}\right)^{\frac{\alpha}{\alpha-d}} x\right): t>0\right) \Longrightarrow\left(\delta\left(Y_{t}^{(1)}\right): t>0\right),
$$

in the sense of convergence of finite dimensional distributions on the space $\mathcal{M}\left(\mathbb{R}^{d}\right)$ equipped with the weak topology. In other words, the scaled solution profile converges to a wandering point mass whose path is given by the process $\left(Y_{t}^{(1)}: t \geq 0\right)$. This formulation of the result is intuitive, but has two drawbacks: first it cannot be improved to convergence on a standard path space, and second it only contains the information that the mass a time $t$ is concentrated in an island of size $o\left((t / \log t)^{\frac{\alpha}{\alpha-d}}\right)$ and hides the fact that this island is indeed a single site.

In order to describe the limit process we need to introduce some notation. Denote by $\Pi$ a Poisson point process on the cone

$$
H^{0}:=\left\{(x, y) \in \mathbb{R}^{d} \times \mathbb{R}: y>-\frac{d}{\alpha-d}\|x\|_{1}\right\}
$$

with intensity measure

$$
\nu(\mathrm{d} x \mathrm{~d} y)=\mathrm{d} x \otimes \frac{\alpha \mathrm{d} y}{\left(y+\frac{d}{\alpha-d}\|x\|_{1}\right)^{\alpha+1}} .
$$

Given this point process, we can define an $\mathbb{R}^{d}$-valued process $Y_{t}^{(1)}$ and an $\mathbb{R}$-valued process $Y_{t}^{(2)}$ in the following way. Fix $t>0$ and define the open cone with tip $(0, z)$ as

$$
\mathcal{C}_{t}(z)=\left\{(x, y) \in \mathbb{R}^{d} \times \mathbb{R}: y+\frac{d}{\alpha-d}\left(1-\frac{1}{t}\right)\|x\|_{1}>z\right\},
$$

and let

$$
\mathcal{C}_{t}=\operatorname{cl} \bigcup_{z>0}\left\{\mathcal{C}_{t}(z): \Pi\left(\mathcal{C}_{t}(z)\right)=0\right\}
$$




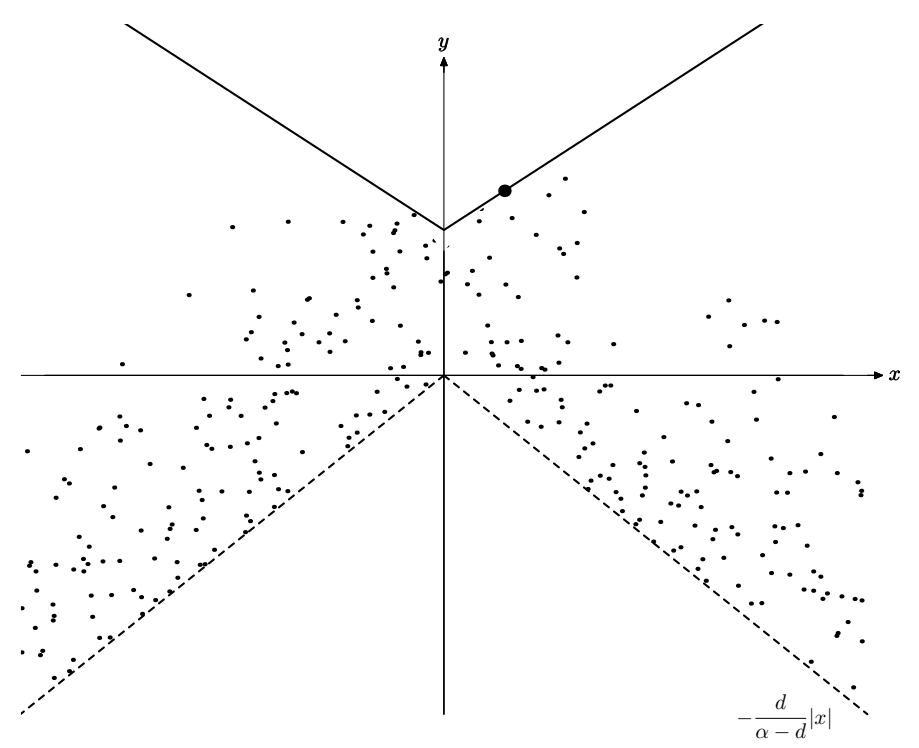

(a) $t<1$.

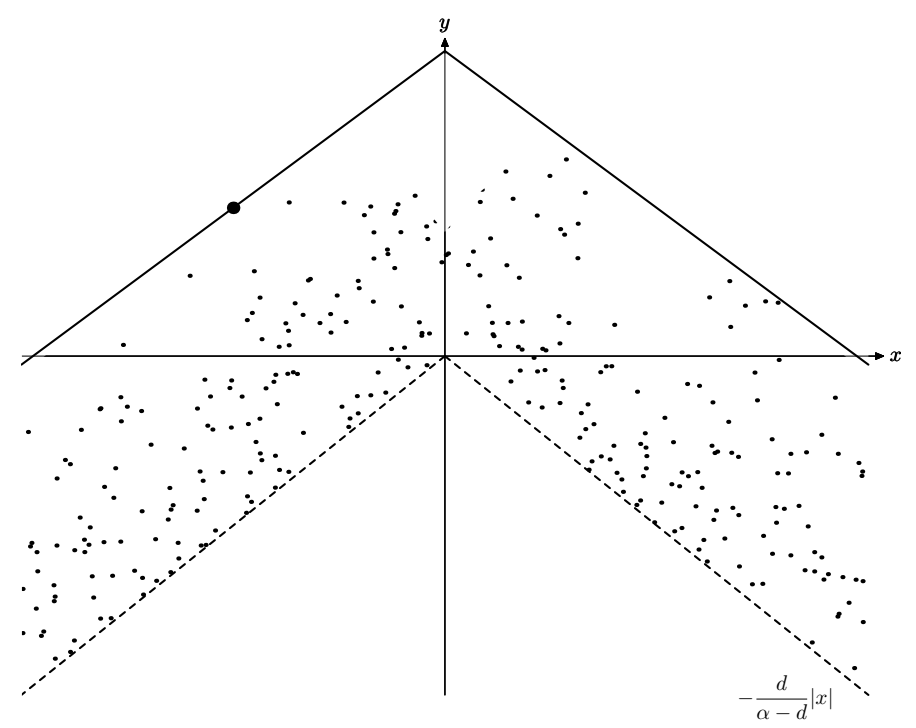

(b) $t>1$.

Figure 1. The definition of the process $\left(Y_{t}^{(1)}, Y_{t}^{(2)}\right)$ in terms of the point process $\Pi$. Note that $t$ parametrises the opening angle of the cone, see (a) for $t<1$ and (b) for $t>1$. 
Informally, $\mathcal{C}_{t}$ is the closure of the first cone $\mathcal{C}_{t}(z)$ that 'touches' the point process as we decrease $z$ from infinity. Since $\mathcal{C}_{t} \cap \Pi$ contains at most two points, we can define $\left(Y_{t}^{(1)}, Y_{t}^{(2)}\right)$ as the point in this intersection whose projection on the first component has the largest $\ell^{1}$-norm, see Figures $1(\mathrm{a})$ and $1(\mathrm{~b})$ for an illustration. The resulting process $\left(\left(Y_{t}^{(1)}, Y_{t}^{(2)}\right): t>0\right)$ is an element of $D(0, \infty)$, the space of càdlàg functions on $(0, \infty)$ taking values in $\mathbb{R}^{d} \times \mathbb{R}$.

Remark 4.3. Time evolution of the process.

(i) $\left(Y_{1}^{(1)}, Y_{1}^{(2)}\right)$ is the 'highest' point of the Poisson point process $\Pi$.

(ii) Given $\left(Y_{t}^{(1)}, Y_{t}^{(2)}\right)$ and $s \geq t$ we consider the surface given by all $(x, y) \in$ $\mathbb{R}^{d} \times \mathbb{R}$ such that

$$
y=Y_{t}^{(2)}-\frac{d}{\alpha-d}\left(1-\frac{1}{s}\right)\left(\|x\|_{1}-\left\|Y_{t}^{(1)}\right\|_{1}\right) .
$$

For $s=t$ there are no points of $\Pi$ above this surface, while $\left(Y_{t}^{(1)}, Y_{t}^{(2)}\right)$ (and possibly one further point) is lying on it. We now increase the parameter $s$ until the surface hits a further point of $\Pi$. At this time $s>t$ the process jumps to this new point $\left(Y_{s}^{(1)}, Y_{s}^{(2)}\right)$. Geometrically, increasing $s$ means opening the cone further keeping the point $\left(Y_{t}^{(1)}, Y_{t}^{(2)}\right)$ on the boundary and moving the tip upwards on the $y$-axis.

(iii) Similarly, given the point $\left(Y_{t}^{(1)}, Y_{t}^{(2)}\right)$ one can go backwards in time by decreasing $s$, or equivalently closing the cone and moving the tip downwards on the $y$-axis. The independence properties of Poisson processes ensure that this procedure yields a process $\left(\left(Y_{t}^{(1)}, Y_{t}^{(2)}\right): t>0\right)$ which is Markovian in both the forward and backward direction. Note however that the projection $\left(Y_{1}^{(1)}: t>0\right)$ is not Markovian (in either time direction).

(iv) An animation of the process $\left(\left(Y_{t}^{(1)}, Y_{t}^{(2)}\right): t>0\right)$ provided by Marcel Ortgiese can be found at http://people.bath.ac.uk/maspm/animation_ageing.pdf.

Remark 4.4. The process which describes the asymptotics of the scaled potential value in the peak,

$$
\left(Y_{t}^{(2)}+\frac{d}{\alpha-d}\left\|Y_{t}^{(1)}\right\|_{1}: t>0\right),
$$

corresponds to the vertical distance of the point $\left(Y_{t}^{(1)}, Y_{t}^{(2)}\right)$ to the boundary of the domain $H_{0}$ given by $y=-\frac{d}{\alpha-d}\|x\|_{1}$, see Figure $2(\mathrm{a})$. The process which describes the asymptotics of the scaled growth rate of the solution,

$$
\left(Y_{t}^{(2)}+\left(1-\frac{1}{t}\right)\left\|Y_{t}^{(1)}\right\|_{1}: t>0\right)
$$

corresponds to the $y$-coordinate of the tip of the cone $\mathcal{C}_{t}$, see Figure 2(b). 


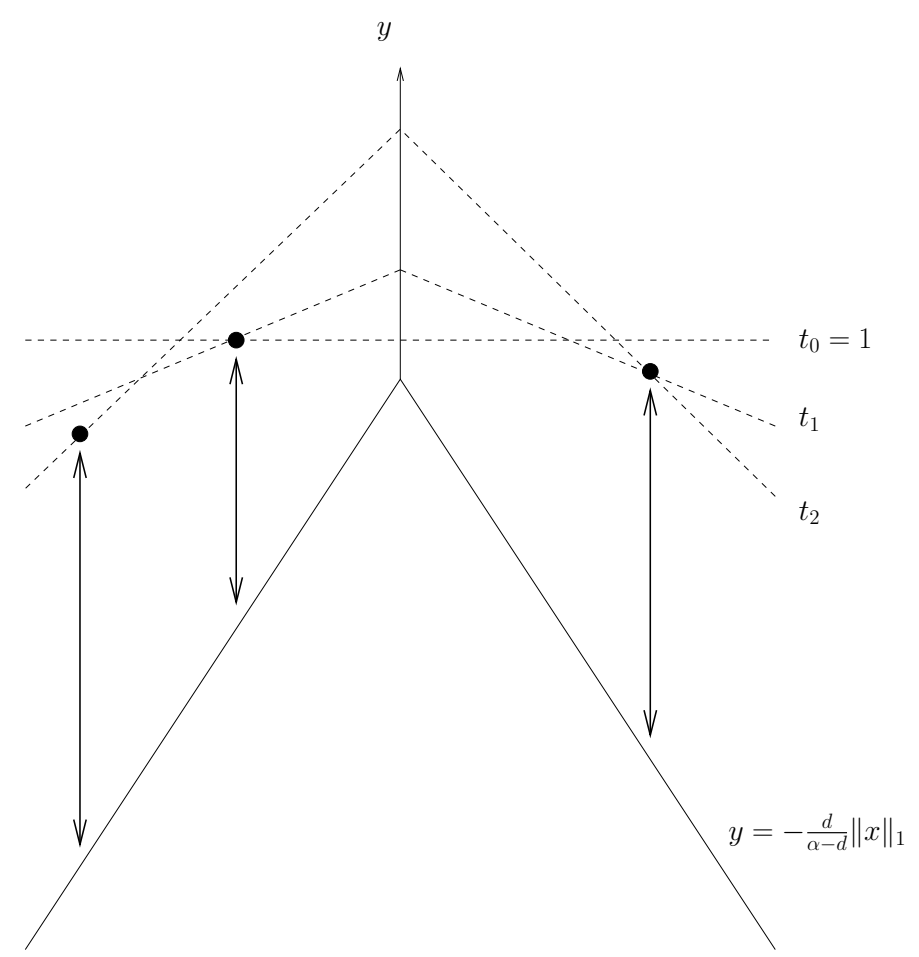

(a) The process describing the potential in the peak.

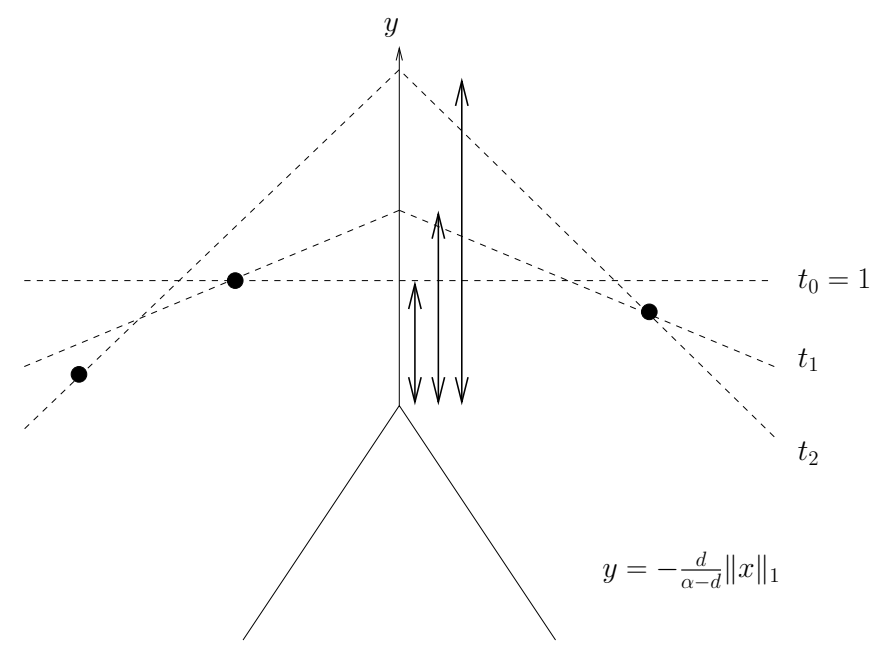

(b) The process describing the growth rate of the solution.

Figure 2. The position of the cone $\mathcal{C}_{t}$ at three times $1=t_{0}<t_{1}<t_{2}$ is indicated by the three dashed contours. The maximal point of the Poisson process is marked by the bold dot on the dashed horizontal line. The two times $t_{1}$ and $t_{2}$ are jump times for the process $\left(Y_{t}^{(1)}: t \geq 1\right)$ with the Poisson points triggering the jumps marked. The vertical positions of the three dots represent the value of this process. (a) The length of the arrows indicate the value of the process $\left(Y_{t}^{(2)}+\frac{d}{\alpha-d}\left\|Y_{t}^{(1)}\right\|_{1}: t>0\right)$ at the three times corresponding to the dots at the end of the arrows. (b) The length of the arrows indicate the value of the process $\left(Y_{t}^{(2)}+\left(1-\frac{1}{t}\right)\left\|Y_{t}^{(1)}\right\|_{1}: t>0\right)$ at the three times, increasing from left to right. 
The proof of this result uses the point process technique developed in [HMS08]. We briefly describe the main idea here. Recall that at most times the peak $X_{t}$ equals the maximiser $Z_{t}$ of the variational problem given by $\Psi_{t}$. We have seen that, in probability,

$$
\frac{1}{T} \log U(T) \sim \max _{z \in \mathbb{Z}^{d}} \Psi_{T}(z)
$$

For $r_{T}=(T / \log T)^{\frac{\alpha}{\alpha-d}}$ and $a_{T}=(T / \log T)^{\frac{d}{\alpha-d}}$ the point process

$$
\Pi_{T}=\sum_{z \in \mathbb{Z}^{d}} \delta_{\left(\frac{z}{r_{T}}, \frac{\Psi_{T}(z)}{a_{T}}\right)},
$$

where $\delta_{x}$ denotes the Dirac measure in $x$, converges to a Poisson point process $\Pi$ with intensity measure $\nu(\mathrm{d} x \mathrm{~d} y)$, as defined in the description of the limiting process. For fixed $t$ and large $T$ we obtain, when $z / r_{T}$ is of constant order,

$$
\frac{\Psi_{t T}(z)}{a_{T}} \approx \frac{\Psi_{T}(z)}{a_{T}}+\frac{d}{\alpha-d}\left(1-\frac{1}{t}\right) \frac{\|z\|_{1}}{r_{T}} .
$$

Note further that

$$
\frac{\xi(z)}{a_{T}} \approx \frac{\Psi_{T}(z)}{a_{T}}+\frac{d}{\alpha-d} \frac{\|z\|_{1}}{r_{T}} .
$$

This allows us to approximate the events of interest with events involving only the point proces $\Pi_{T}$. Informally, we obtain

$$
\begin{aligned}
& P\left\{\frac{Z_{t T}}{r_{T}} \in A, \frac{\xi\left(Z_{t T}\right)}{a_{T}} \in B\right\} \\
& \approx \iint_{x \in A, y+\frac{d}{\alpha-d} x \in B} P\left\{\Pi_{T}(\mathrm{~d} x \mathrm{~d} y)>0,\right. \\
& \left.\quad \Pi_{T}\left\{(\bar{x}, \bar{y}): \bar{y}-y>\frac{d}{\alpha-d}\left(1-\frac{1}{t}\right)\left(\|x\|_{1}-\|\bar{x}\|_{1}\right)\right\}=0\right\},
\end{aligned}
$$

where the first line of conditions on the right means that there is a site $z / r_{T} \in A$ with $\Psi_{T}(z) / a_{T}=y$ and $\xi(z) / a_{T} \in B$, and the second line means that $\Psi_{t T}(z)$ is not surpassed by $\Psi_{t T}(\bar{z})$ for any other site $\bar{x}=\bar{z} / r_{T}$. We can now use the convergence of $\Pi_{T}$ to $\Pi$ inside the formula to give the limit theorem for the finite-dimensional distributions of

$$
\left(\left(\frac{X_{t T}}{r_{T}}, \frac{\xi\left(X_{t T}\right)}{a_{T}}\right): t>0\right) \text {. }
$$

Checking a tightness criterion in Skorokhod space completes the argument.

\section{Ageing in the parabolic Anderson model}

In a physical system which changes over time we are naturally interested in the time scales in which we experience significant changes of the system. If the system has randomness we may, for example, try to find a function $s(t)$ such that the 
probability that the state at time $t$ remains unchanged up to time $t+s(t)$ remains bounded from zero and one, as $t \uparrow \infty$. We may say that the system exhibits ageing if $s(t)$ goes to infinity as $t \uparrow \infty$, while in typical cases of ageing we even observe a linear dependence of $s(t)$ on $t$. Hence, as time goes on, in an ageing system changes become less likely and the typical time scales of the system are increasing. Therefore, ageing can be associated to the existence of infinitely many time-scales that are inherently relevant to the system. This is in marked contrast to metastable systems, which are characterised by a finite number of well separated time-scales, corresponding to the lifetimes of different metastable states.

Ageing has been the subject of extensive research. Some interesting papers exhibiting the ageing phenomenon include the case of spherical spin glasses [BDG01], the random energy model with Glauber dynamics [BBG03] and interacting diffusions [DD07]. The bulk of the research however is on very simple trap models which give a phenomenological description of a particle moving in an energy landscape getting trapped in deeper and deeper energy wells. Interest for trap models in the mathematical community was created through the pioneering work of [FIN02] and [BCM06], and a survey is provided in the lecture notes of [BC06]. Recent work of Ben Arous and Cerný [BC08] shows that in the case of trap models ageing is naturally linked with the arcsine law for stable subordinators, and this connection is believed to be of a universal nature.

Coming back to the parabolic Anderson model with Pareto tails, we can conjecture on the basis of the scaling limit theorem that the system exhibits some form of ageing: doubling the length of the observation window asymptotically doubles the length of periods of near constancy of the solution profile. However, the statement of the scaling limit theorem is not strong enough to verify a full ageing result, which can be obtained by other means.

Theorem 5.1 (Ageing in probability, Theorem 1.1 in [MOS09].). For any $\theta>0$ there exists $0<I(\theta)<1$ such that, for all $0<\varepsilon<\frac{1}{2}$,

$$
\begin{aligned}
\lim _{t \uparrow \infty} P & \left\{\sup _{z \in \mathbb{R}^{d}} \sup _{s \in[t, t+t \theta)]}|v(t, z)-v(s, z)|<\varepsilon\right\} \\
& =\lim _{t \uparrow \infty} P\left\{\sup _{z \in \mathbb{R}^{d}}|v(t, z)-v(t+t \theta, z)|<\varepsilon\right\} \\
& =I(\theta) .
\end{aligned}
$$

Remark 5.2. As discussed in [BC05] in trap models it is often the case that a particle is in the same state at times $t$ and $t+s(t)$ but has left this state briefly several times during the interval $[t, t+s(t)]$. This can lead to different relevant scales for the two limits above. For the parabolic Anderson model this is is not the case, the profile never returns to an earlier state.

Remark 5.3. The constant $I(\theta) \in(0,1)$ can be given explicitly in terms of an integral. The most interesting fact is that it does not come from a generalised 
arcsine law as in the paradigm cases described in [BC08]. We can also describe its tails at infinity and zero as

$$
I(\theta) \sim C \theta^{-d} \text { as } \theta \uparrow \infty, \quad \text { and } \quad 1-I(\theta) \sim c \theta \text { as } \theta \downarrow 0,
$$

for explicit constants $0<c, C<\infty$.

Let us briefly discuss the proof of Theorem 5.1. We first show that

$$
\begin{gathered}
\lim _{t \uparrow \infty} P\left\{\sup _{z \in \mathbb{R}^{d}} \sup _{s \in[t, t+t \theta]}|v(t, z)-v(s, z)|<\varepsilon\right\} \\
=\lim _{t \uparrow \infty} P\left\{Z_{t}=Z_{t+t \theta}\right\},
\end{gathered}
$$

where $Z_{t}$ can be taken to be the maximiser in the variational problem given by $\Psi_{t}$. To discuss the limit on the right hand side we again approximate the probability on the right hand side in terms of the point process $\Pi_{t}$. We are able to write

$$
\frac{\Psi_{t+\theta t}(z)}{a_{t}}=\frac{\Psi_{t}(z)}{a_{t}}+\frac{\theta}{1+\theta} \frac{d}{\alpha-d} \frac{|z|}{r_{t}}+\text { error }
$$

where the error can be suitably controlled. Hence (in symbolic notation)

$$
\begin{aligned}
& P\left\{Z_{t}=Z_{t+t \theta}\right\} \\
& \quad \approx \iint P\left\{\Pi_{t}(\delta x \delta y)>0, \Pi_{t}\{(\bar{x}, \bar{y}): \bar{y}>y\}=0,\right. \\
& \left.\quad \Pi_{t}\left\{(\bar{x}, \bar{y}):|\bar{x}|>|x| \text { and } \bar{y}>y-\frac{d}{\alpha-d} \frac{\theta}{1+\theta}(|\bar{x}|-|x|)\right\}=0\right\},
\end{aligned}
$$

where the first line of conditions on the right means that $x$ is a maximizer of $\Psi_{t}$ with maximum $y$, and the second line means that $x$ is also a maximizer of $\Psi_{t+\theta t}$. As $t \uparrow \infty$ the point process $\Pi_{t}$ is replaced by $\Pi$ and we can evaluate the probability. and complete the proof.

It is more difficult to come to a notion of ageing in an almost-sure sense, which is observable from a typical trajectory of the solution. Roughly speaking, given the state of the system at some time $t$, we may ask for the maximal time $R(t)$ such that the system is still in the same state at time $t+R(t)$. This 'residual lifetime function' - to borrow a terminology from renewal theory - hits zero whenever there is a change of state. To find a meaningful notion of the time scale in which we experience a change we would therefore ask for a characterisation of the upper envelopes of the residual lifetime function.

To make this plan concrete recall that $\left(X_{t}: t \geq 0\right)$ is the process of peaks of the solution. Define the residual lifetime function by

$$
R(t)=\sup \left\{s \geq 0: X_{t}=X_{t+s}\right\},
$$


for $t \geq 0$. Roughly speaking, $R(t)$ is the waiting time, at time $t$, until the next change of peak, see the schematic picture in Figure 3. We have shown in Theorem 5.1 that the law of $R(t) / t$ converges to the law given by the distribution function $1-I$. In the following theorem, we describe the smallest asymptotic upper envelope for the process $(R(t): t \geq 0)$.

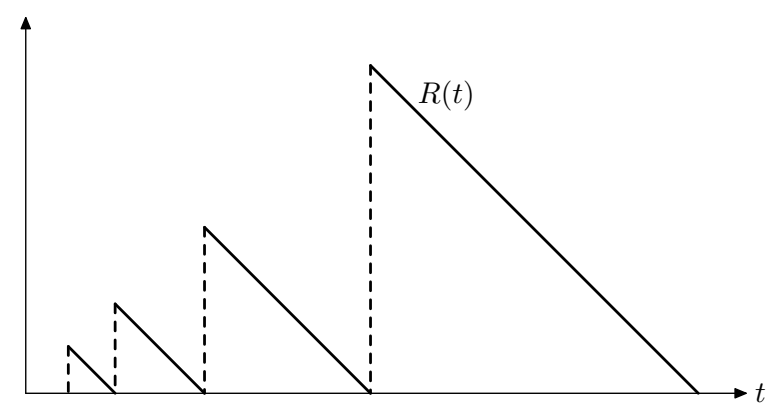

Figure 3. A schematic representation of the remaining lifetime function $R$.

Theorem 5.4 (Almost sure ageing, Theorem 1.2 in [MOS09]). For any nondecreasing function $h:(0, \infty) \rightarrow(0, \infty)$ we have, almost surely,

$$
\limsup _{t \uparrow \infty} \frac{R(t)}{t h(t)}= \begin{cases}0 & \text { if } \int_{1}^{\infty} \frac{\mathrm{d} t}{t h(t)^{d}}<\infty, \\ \infty & \text { if } \int_{1}^{\infty} \frac{\mathrm{d} t}{t h(t)^{d}}=\infty .\end{cases}
$$

The proof of Theorem 5.4 is technically more involved, because we can no longer benefit from the point process approach and have to do significant parts of the argument from first principles. We consider events

$$
P\left\{\frac{R(t)}{t} \geq \theta_{t}\right\} \approx P\left\{Z_{t}=Z_{t+t \theta_{t}}\right\}
$$

for $\theta_{t} \uparrow \infty$. We significantly refine the argument leading to Theorem 5.1 and replace the convergence of $P\left\{Z_{t}=Z_{t+t \theta}\right\}$ by a moderate deviation statement: For $\theta_{t} \uparrow \infty$ not too fast we show that

$$
P\left\{Z_{t}=Z_{t+t \theta_{t}}\right\} \sim C \theta_{t}^{-d}
$$

for a suitable constant $C>0$. Then, if $\varphi(t)=t h(t)$, this allows us to show that, for any $\varepsilon>0$, the series $\sum_{n} P\left\{R\left(e^{n}\right) \geq \varepsilon \varphi\left(e^{n}\right)\right\}$ converges if $\sum_{n} h\left(e^{n}\right)^{-d}$ converges, which is essentially equivalent to $\int h(t)^{-d} \mathrm{~d} t / t<\infty$. By Borel-Cantelli we get that

$$
\limsup _{n \rightarrow \infty} \frac{R\left(e^{n}\right)}{\varphi\left(e^{n}\right)}=0,
$$

which implies the upper bound in Theorem 5.4. The lower bound follows using a more delicate second moment estimate. 


\section{Conclusion}

The aim of this project was to study the possible effects of a highly irregular potential on a diffusion on a $d$-dimensional lattice. By modeling the potential as a spatially independent, identically distributed random field with polynomial tails we have seen that the diffusion shows interesting extreme behaviour, in particular

- the growth rate of the total mass is asymptotically random,

- the solution is asymptotically concentrated in a single point at most times,

- this point goes to infinity at superlinear speed,

- the solution is asymptotically concentrated in two points at all times,

- the system exhibits ageing behaviour.

In the proofs we combine a very fine analysis of the random walk paths contributing in the Feynman-Kac formula with extreme value theory for the random field.

Acknowledgement: I would like to thank all my collaborators in the various stages of this project, namely Remco van der Hofstad, Wolfgang König, Hubert Lacoin, Marcel Ortgiese and Nadia Sidorova, for many enlightening disussions and for all the great ideas they contributed. It was a privilege to work with them. I am also grateful for the support of EPSRC through an Advanced Research Fellowship.

\section{References}

[An58] P.W. Anderson, Absence of diffusion in certain random lattices. Phys. Rev. 109, 1492-1505 (1958).

[BBG03] G. Ben Arous, A. Bovier and V. Gayrard. Glauber dynamics of the random energy model. II. Aging below the critical temperature. Comm. Math. Phys. 236, 1-54 (2003).

[BC06] G. Ben Arous and J. Černý. Dynamics of trap models. In Ecole d'Eté de Physique des Houches LXXXIII, "Mathematical Statistical Physics", pp. 331-394. Amsterdam: North-Holland (2006).

[BC05] G. Ben Arous and J. Černý. Bouchaud's model exhibits two different aging regimes in dimension one, Ann. Appl. Probab. 15, 1161-1192 (2005).

[BC08] G. Ben Arous and J. ČERnÝ. The arcsine law as a universal aging scheme for trap models. Comm. Pure Appl. Math. 61, 289-329 (2008). 
[BCM06] G. Ben Arous, J. Černý and T. Mountford. Aging in two-dimensional Bouchaud's model. Probab. Theory and Relat. Fields 134, 1-43 (2006).

[BDG01] G. Ben Arous, A. Dembo and A. Guionnet. Aging of spherical spin glasses. Probab. Theory and Relat. Fields 120, 1-67 (2001).

[BMr07] G. Ben Arous, S. Molchanov and A. Ramirez, Transition asymptotics for reaction-diffusion in random media. In Probability and Mathematical Physics: A Volume in Honor of Stanislav Molchanov, AMS/CRM, 42, 140 (2007).

[BK01] M. Biskup and W. KöNIG. Long-time tails for the parabolic Anderson model with bounded potential. Ann. Probab. 29, 636-682 (2001).

[BF05] A. Bovier and A. Faggionato. Spectral characterization of aging: the REM-like trap model. Ann. Appl. Probab., 15, 1997-2037, (2005).

[CM94] R. Carmona and S.A. Molchanov, Parabolic Anderson problem and intermittency. Mem. Amer. Math. Soc. 108, no. 518, (1994).

[DD07] A. Dembo and J.-D. Deuschel, Aging for interacting diffusion processes. Ann. Inst. H. Poincaré Probab. Statist. 43, 461-480 (2007).

[D08] A. Drewitz. Lyapunov exponents for the one-dimensional parabolic Anderson model with drift. Electron. J. Probab., 13, 2283-2336 (2008).

[Fin02] L. R. G. Fontes, M. Isopi, and C. M. Newman. Random walks with strongly inhomogeneous rates and singular diffusions: convergence, localization and aging in one dimension. Ann. Probab. 30, 579-604 (2002).

[FG92] K. Fleischmann and A. Greven, Localization and selection in a mean field branching random walk in a random environment. Ann. Probab. 20, 2141-2163 (1992).

[FM90] K. Fleischmann and S. Molchanov, Exact asymptotics in a mean field model with random potential. Probab. Theory Relat. Fields 86, 239-251 (1990).

[GHM07] J. Gärtner, F. Den Hollander and G. Mailllard, Intermittency on catalysts: symmetric exclusion. Electron. J. Probab. 12, 516-573 (2007).

[GHM08] J. GÄRTner, F. DEN Hollander and G. Mailllard, Intermittency on catalysts. In: J. Blath, P. Mörters and M. Scheutzow (Eds.), Trends in Stochastic Analysis, pp. 235-248, Cambridge University Press, Cambridge 2008.

[GK05] J. GäRTnER and W. KöNig, The parabolic Anderson model. In: J.-D. Deuschel and A. Greven (Eds.), Interacting Stochastic Systems, pp. 153-179, Springer, Berlin 2005.

[GKM07] J. Gärtner, W. KÖNIG and S. Molchanov, Geometric characterisation of intermittency in the parabolic Anderson model. Ann. Probab. 35, 439-499 (2007). 
[GM90] J. GÄRTner and S. Molchanov, Parabolic problems for the Anderson model. I. Intermittency and related topics. Commun. Math. Phys. 132, 613655 (1990).

[GM98] J. GÄRTner and S. Molchanov, Parabolic problems for the Anderson model. II. Second-order asymptotics and structure of high peaks. Probab. Theory Relat. Fields 111, 17-55 (1998).

[HKM06] R. VAN DER HOFstad, W. KÖNIG and P. MÖrTERs, The universality classes in the parabolic Anderson model. Commun. Math. Phys. 267, 307353 (2006).

[HMS08] R. van der Hofstad, P. MÖrters and N. Sidorova, Weak and almost sure limits for the parabolic Anderson model with heavy tailed potentials. Ann. Appl. Probab. 18, 2450-2494 (2008).

[KLMS09] W. König, H. Lacoin, P. Mörters and N. Sidorova, A two cities theorem for the parabolic Anderson model. Ann. Probab. 37, 347-392 (2009).

[KMS06] W. König, P. Mörters and N. Sidorova, Complete localisation in the parabolic Anderson model with Pareto-distributed potential. Preprint at math.PR/0608544 .

[MOS09] P. Mörters, M. Ortgiese and N. Sidorova, Ageing in the parabolic Anderson model. Preprint at arXiv:0910.5613.

[Mo94] S. Molchanov, Lectures on random media. In: D. Bakry, R.D. Gill, and S. Molchanov, Lectures on Probability Theory, Ecole d'Eté de Probabilités de Saint-Flour XXII-1992, LNM 1581, pp. 242-411. Springer, Berlin 1994.

[Sz98] A.-S. Sznitman, Brownian motion, Obstacles and Random Media. Springer, Berlin 1998.

[ZMRS87] Ya. B. Zel'Dovitch, S.A. Molchanov, S.A. Ruzmaikin, and D.D. Sokolov, Intermittency in random media. Sov. Phys. Uzpekhi. 30:5, 353369 (1987).

Peter Mörters, Department of Mathematical Sciences, University of Bath, Bath BA2 7AY, United Kingdom

E-mail: maspm@bath.ac.uk 